\title{
Fabrication of a tungsten master stamp using self-ordered porous alumina
}

\author{
Jinsub Choi ${ }^{1,3}$, Young-Bae Park ${ }^{2}$ and Axel Scherer ${ }^{1}$ \\ ${ }^{1}$ Nanofabrication group, Division of Engineering and Applied Physics, California Institute of \\ Technology, Pasadena, CA 91125, USA \\ 2 Thomas J Watson Laboratory of Applied Physics, California Institute of Technology, \\ Pasadena, CA 91125, USA \\ E-mail: jinsub@kicet.re.kr
}

Received 3 May 2005, in final form 25 May 2005

Published 6 July 2005

Online at stacks.iop.org/Nano/16/1655

\begin{abstract}
We describe the preparation of a tungsten pillar nanoimprint stamp without the use of lithography and etching techniques. Structures with heights of $15 \mathrm{~nm}$ were prepared on the basis of self-ordered porous alumina templates and this was followed by DC sputtering of tungsten. The stamp was successfully used to prepare an aluminium surface to obtain highly ordered porous anodic alumina films after a single anodization step. The preparation efficiency for highly ordered porous alumina was dramatically improved as compared to the more conventional two-step anodization-strip-anodization method, as a sacrificial layer with a thickness of a few hundred micrometres was not required. In addition, by fractal calculations, we have evaluated the degree of ordering of the asperities on the nanoimprint master stamp.
\end{abstract}

(Some figures in this article are in colour only in the electronic version)

\section{Introduction}

In the past few years, the synthesis of nanotemplates with a uniform size and depth has received great attention as an important route in the fabrication of nanowires and nanotubes [1-3]. Among these, self-ordered porous alumina templates with a pore size controllable from $20 \mathrm{~nm}$ up to $400 \mathrm{~nm}$ have been intensely investigated since their preparation process is cost-effective and very highly reproducible $[4,5]$. However, the self-ordering of pore arrays without a nucleation step to define ordered pore domains takes a long time (usually, over one day). Typically, a two-step anodization is required in order to obtain highly ordered porous alumina with straight pore channels. First an oxide with a thickness of a few hundred micrometres is formed by anodization. This sacrificial anodized alumina layer is then removed, leaving small dimples on the aluminium surface. Self-ordered porous alumina results after a second anodization step which uses the dimples as pore nuclei.

3 Author to whom any correspondence should be addressed. Present address: Biomaterials Laboratory, Nanomaterials Application Division, Korea Institute of Ceramic Engineering and Technology, 233-5, Gasan-Dong, GuemcheonGu, Seoul, 153-801, Korea.
Recently, alternative methods for defining well-ordered porous anodic layers have been developed to avoid consuming a thick sacrificial aluminium layer. These techniques of pore nucleation have been developed on the basis of the formation of prestructures acting as seeds for the electrochemical pore growth [6-12]. E-beam lithography, focused-ion beam lithography, and nanoimprint lithography can allow us effectively to emboss the prepattern so that monodomain porous alumina can be prepared over a large area. Nanoimprint lithography has several advantages in terms of efficiency in preparing highly ordered porous alumina. After a nanoimprint master stamp is fabricated by using a sequence of lithographic device fabrication processes, the stamp is reusable and can produce many highly ordered replica patterns on aluminium surfaces [6,9]. If the thickness for the sacrificial layer is limited to a few micrometres, as in the case for a thin aluminium film prepared through vacuum deposition, nanoimprint lithography is required for the preparation of ordered porous alumina $[13,14]$. Moreover, it was demonstrated that successive nanoimprint lithography processes can create complicated patterns which cannot be easily fabricated by conventional lithography [15].

Although the merits of nanoimprint pore nucleation are well known, only a few groups have successfully applied the 
(a)

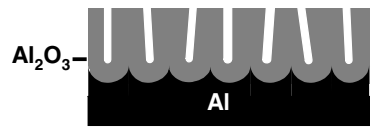

(b)

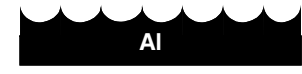

(c)

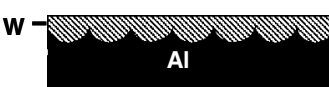

(d)

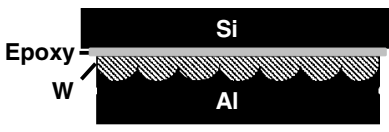

(e)

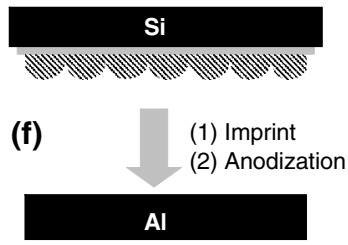

Figure 1. Schematic diagram of the preparation of the tungsten nanoimprint stamp: (a) anodization of aluminium for two days, (b) removal of the first formed oxide, (c) deposition of tungsten by DC sputtering, (d) bonding with a Si substrate, (e) selective removal of the aluminium substrate, and (f) nanoimprint lithography using the prepared tungsten master stamp and successive anodization.

method for the synthesis of ordered porous materials, as the construction of a master stamp requires complicated nanofabrication techniques. Therefore nanoimprint lithography techniques that use self-assembled latex beads with a various diameters have been studied for the preparation of a hexagonal pattern on an aluminium surface for highly ordered porous alumina structures out of the self-ordering regimes [16].

In this study, we show the preparation of a novel nanoimprint stamp consisting of tungsten dots without the need for sophisticated device fabrication procedures. The stamp is an inorganic replication mould of the aluminium surface with hexagonally arranged corrugation. In addition, we will evaluate the degree of ordering of nanodots of the tungsten master stamp by using fractal dimension calculations in order to demonstrate that the tungsten master stamp is immediately applicable for the preparation of highly ordered porous alumina.

\section{Experimental details}

The preparation procedure of tungsten nanoimprint stamp is outlined in figure 1 . The fabrication of highly ordered alumina pore arrays has been reported elsewhere in greater detail $[4,5]$. Electrochemically polished aluminium foil with a diameter of $15 \mathrm{~mm}$ is anodized at $40 \mathrm{~V}$ in $0.3 \mathrm{M}$ oxalic acid at $5^{\circ} \mathrm{C}$ for two days (figure 1(a)). The first formed oxide with a thickness of around $100 \mu \mathrm{m}$ is selectively removed in a mixture of chromic acid $(6 \mathrm{wt} \%)$ and phosphoric acid $(2 \mathrm{wt} \%)$ at $60^{\circ} \mathrm{C}$ for $6 \mathrm{~h}$ (figure 1(b)), resulting in a fresh aluminium substrate with the hexagonally arranged corrugation. Afterwards, a tungsten layer with a thickness of $100 \mathrm{~nm}$ is deposited on the corrugation by DC sputtering (figure 1(c)). The power, time, mass flow rate of $\mathrm{Ar}$, and pressure for the DC sputtering used in this study are $60 \mathrm{~W}, 5 \mathrm{~min}, 20 \mathrm{sccm}$, and $5 \mathrm{mTorr}$, respectively. After a Si substrate with a thickness of $500 \mu \mathrm{m}$ is bonded on the tungsten layer with epoxy glue (figure $1(\mathrm{~d})$ ), the aluminium foil is selectively dissolved in a mixture of $3.4 \mathrm{~g} \mathrm{CuCl}_{2} \cdot 6 \mathrm{H}_{2} \mathrm{O}$, $100 \mathrm{ml} \mathrm{HCl}\left(35 \%\right.$ ) and $100 \mathrm{ml} \mathrm{H}_{2} \mathrm{O}$ (figure 1(e)).

A pneumatic oil press is used for nanoimprint lithography on an $\mathrm{Al}$ substrate (figure 1(f)). The pressure typically used

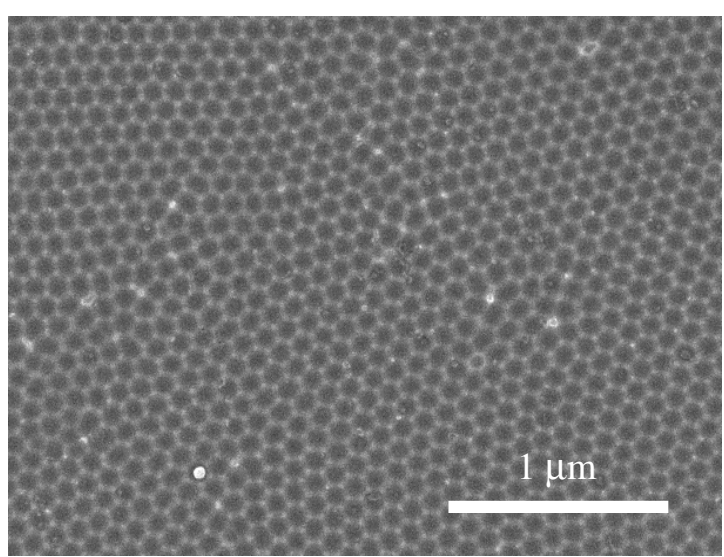

Figure 2. SEM image of the corrugation surface of aluminium which is obtained after removing the first formed oxide (corresponding to figure 1(b)).

Table 1. Vickers hardness of several ceramics and metals.

\begin{tabular}{llllllll}
\hline & $\mathrm{SiC}$ & $\mathrm{Si}_{3} \mathrm{~N}_{4}$ & $\mathrm{~W}$ & $\mathrm{Ti}$ & $\mathrm{Pt}$ & $\mathrm{Au}$ & $\mathrm{Al}$ \\
\hline $\begin{array}{l}\text { Hardness } \\
\text { (Vickers, HV) }\end{array}$ & 2000 & 1600 & 310 & 60 & 40 & 25 & 15 \\
\hline
\end{tabular}

is $15 \mathrm{kN} \mathrm{cm}^{-2}$, which is three times higher than that using the pyramidal stamp [9]. Then, the nanoimprinted aluminium is anodized in self-ordering regimes at $40 \mathrm{~V}$. The prepared structures are characterized by scanning electron microscopy (SEM) and atomic force microscopy (AFM). For evaluations of the degree of pore ordering, the fractal dimension and lateral correlation length are obtained with a height-height correlation function using the MATLAB code.

\section{Results and discussion}

As mentioned in the introduction section above, the preparation of master stamps made of $\mathrm{SiC}$ and $\mathrm{Si}_{3} \mathrm{~N}_{4}$ has been demonstrated by several groups [6,9]. Since the ceramics exhibit a strong hardness compared to metals (see table 1) [17], the ceramic stamps have been successfully used for nanoimprint lithography on ductile metals such as $\mathrm{Al}$ and Ti $[6,9,18]$. However, the process for making the ceramic stamps is quite complicated because of, ironically, the hardness. For instance, a PECVD (plasma enhanced chemical vapour deposition) step and a dry etching are required for making the $\mathrm{Si}_{3} \mathrm{~N}_{4}$ stamp and the $\mathrm{SiC}$ stamp, respectively. In the case of the preparation of the highly ordered porous alumina, extremely hard materials are not required, since the hardness of aluminium is only $15 \mathrm{HV}$. Thus, tungsten is a good candidate for making the nanoimprint master stamp because it can be easily deposited on a desired substrate by DC sputtering and is one of the hardest metals (according to table 1, it is at least 20 times harder than aluminium). This hardness is the primary reason that tungsten was chosen in this study.

Figure 2 shows the surface of aluminium with hexagonally arranged patterns, corresponding to figure 1(b). The selfordered dimples have several domains with the same direction of ordering, which are observed in the self-ordered porous 
Fabrication of a tungsten master stamp using self-ordered porous alumina

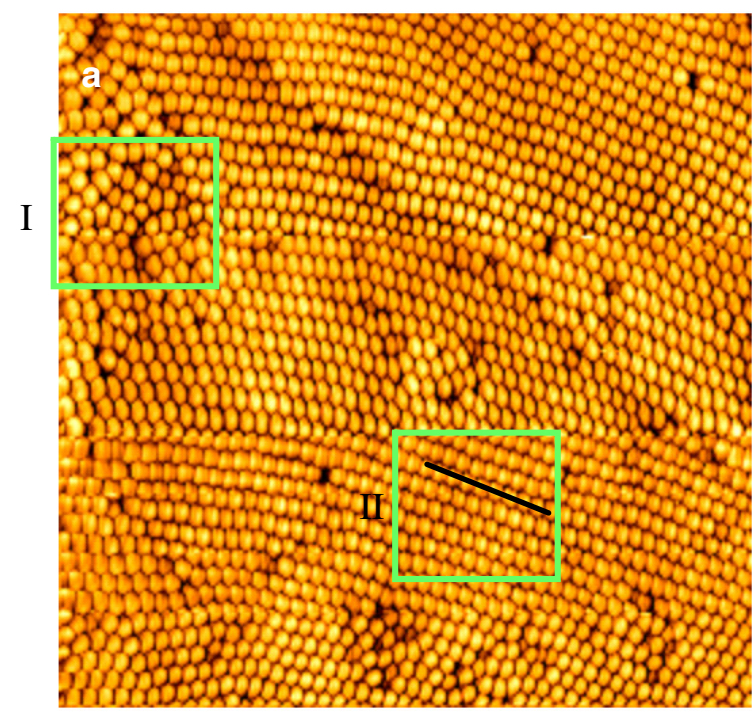

\section{b}
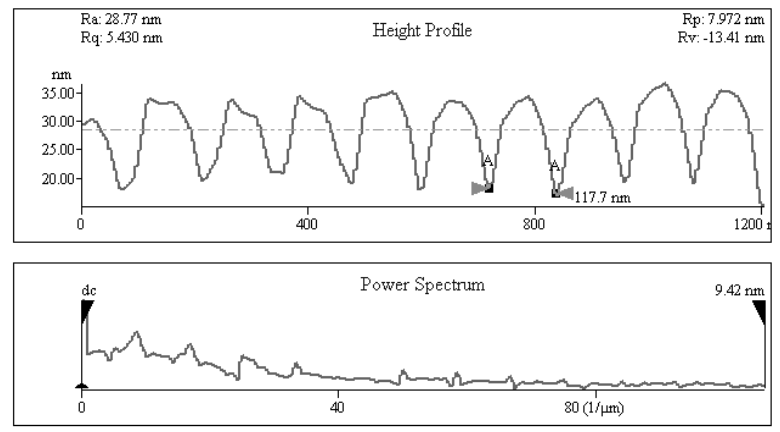

Figure 3. (a) AFM topography of the tungsten nanoimprint stamp. (b) The height profile of the region indicated by II in (a). I and II in (a) represent disordered and ordered regions, respectively.

alumina. The mean depth of dimples was measured as $25 \mathrm{~nm}$, which is in good agreement with the previous measurement [19].

Figure 3 is the AFM topography of a master stamp consisted of the tungsten nanodots, corresponding to figure 1(e). Even though defects appear at some points, overall, highly ordered tungsten dots are observed in the figure 3(a). Since the thin aluminium substrate is very flexible and easily deformed in the course of the preparation (figure 1(b)), we speculate that the disordered area indicated by I in figure 3(a) originated from a local deformation of the corrugation surface. Thus, the use of thicker aluminium as a starting substrate is preferred to avoid any deformation. The profile of the nanodots was measured in the highly ordered area (II in figure 3(a)), indicating that the mean height of the tungsten dots is around $15 \mathrm{~nm}$. Since a minimum depth of $3 \mathrm{~nm}$ is required for the preparation of highly ordered porous alumina [20], we believe that the height of the master stamp was sufficient. The hexagonal patterns on the tungsten master stamp were transferred onto an aluminium substrate under a high pressure, considering that the dot shape is not as sharp for our technique as that generally used for a pyramidal stamp made of $\mathrm{Si}_{3} \mathrm{~N}_{4}$. For example, if $5 \mathrm{kN} \mathrm{cm}^{-2}$, the optimized pressure for the pyramidal stamp, is used, we only obtain partially ordered
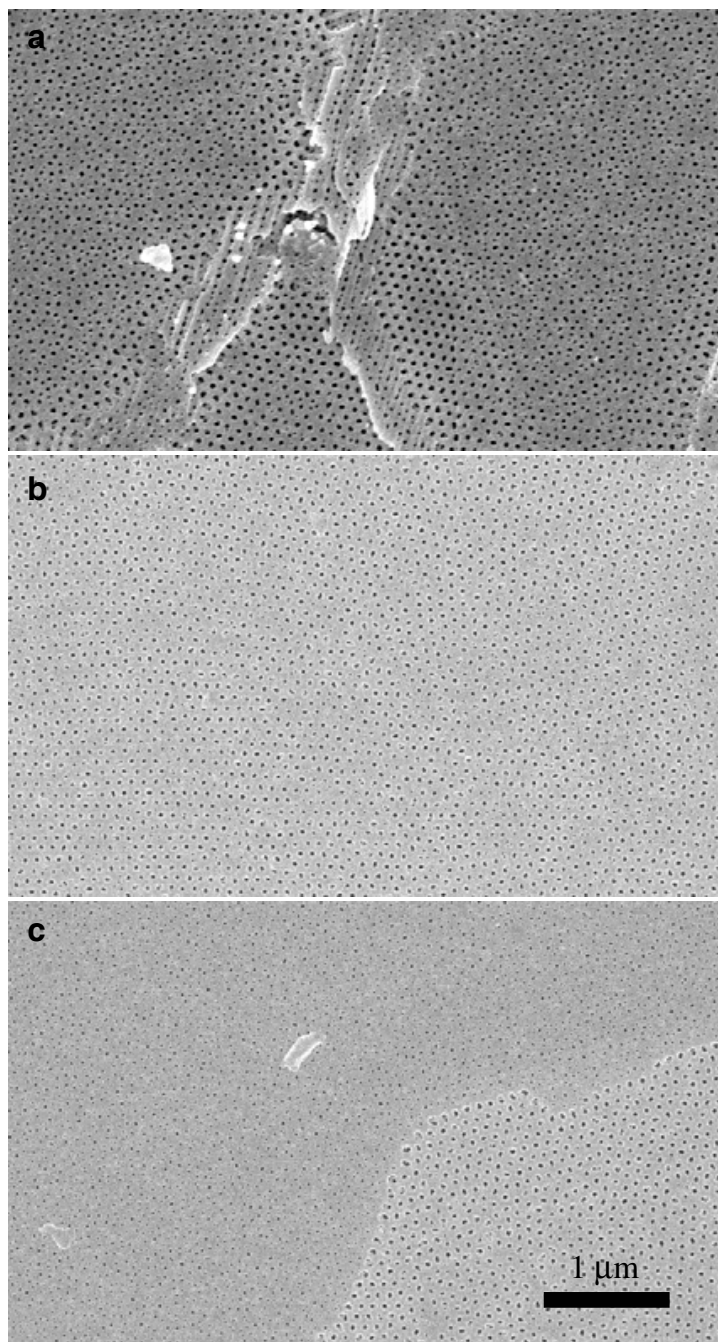

Figure 4. SEM images of porous alumina arrays obtained by anodization of $\mathrm{Al}$ nanoimprinted under the pressure of

(a) $5 \mathrm{kN} \mathrm{cm}^{-2}$ and ((b), (c)) $15 \mathrm{kN} \mathrm{cm}^{-2}$. In (c), one can observe the obvious difference between the anodization of the imprinted area and that of the not-imprinted area.

porous alumina on the ridge of the rough aluminium surface as shown in figure 4(a). When the pressure is increased to 3 times, we can achieve highly ordered porous alumina over a large area with the tungsten stamp (figure 4(b)). Note that a higher pressure not only makes the prepatterns but also flattens the surface. Both actions are needed to increase the degree of ordering of porous alumina [21].

We can see the difference between the nanoimprinted area and not-nanoimprinted area more clearly at the boundary of the stamp when it is anodized (figure 4(c)). We can observe that an arrangement of pore arrays is dramatically improved in the imprinted area, and there is an obvious difference of density of the pores in the two regions. This demonstrates that the tungsten nanoimprint stamp can be successfully applied to obtain highly ordered porous alumina by a single anodization step.

The degree of ordering of nanodots on the master stamp was examined by fast Fourier transformation (FFT) of the AFM images for different scanning regions. In the FFT images, the 

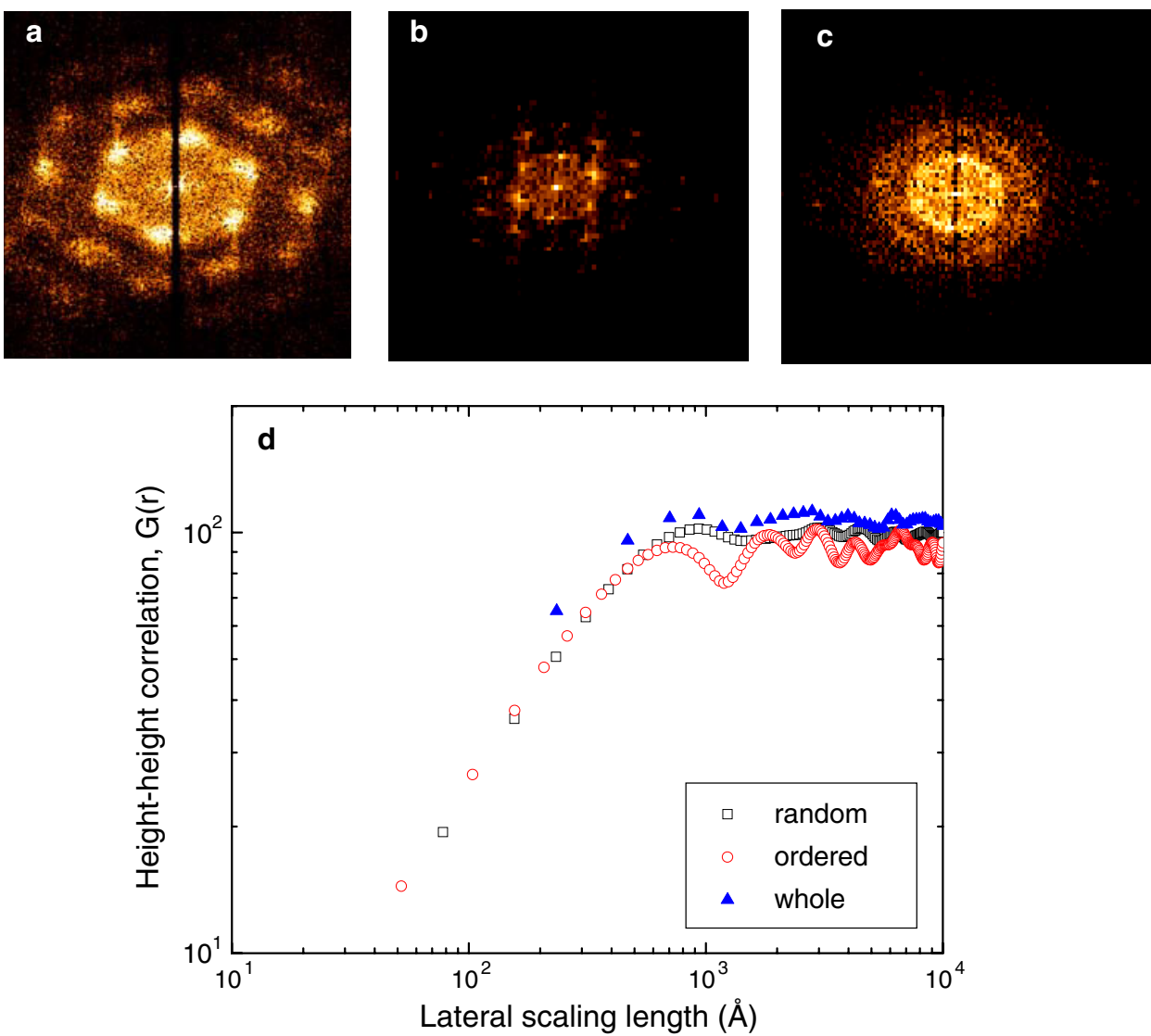

Figure 5. Fast Fourier transformation (FFT) of AFM images for different scanning regions: (a) entire area, (b) ordered region (the region II in figures 3(a)), and (c) disordered region (the region I in figure 3(a)). (d) The surface height-height correlation function obtained from AFM measurement in the three corresponding regions.

ordered region shows hexagonal surface structure while the disordered region shows a ring shape (figures 5(a)-(c)).

Furthermore, numerical evaluations of the ordered region, disordered region and whole area were performed using the fractal scaling law. With parameters obtained from the fractal calculation, one can simulate an imaginary ideal surface. Correlation data were obtained from selected scanning ranges with $256 \times 256$ pixels. The surface height-height correlation function, $G(R)$, is defined as $G(R)=\left\langle\left[h\left(x_{2}, y_{2}\right)^{2}-\right.\right.$ $\left.\left.h\left(x_{1}, y_{1}\right)^{2}\right]\right\rangle$, where $R=\left[\left(x_{2}-x_{1}\right)^{2}+\left(y_{2}-y_{1}\right)^{2}\right]^{1 / 2}$, and $\langle\cdots\rangle$ indicates an average over the entire $x-y$ plane $[22,23]$. The $G(r)$ function represents the surface morphology as self-affine (invariant under an anisotropic scaling length) or self-similar ('mounded' with a regular mound structure) surface, giving the values of the spatial roughness parameter. The calculation of $G(R)$ was performed along the tip scan $x$ and $y$ directions. Such morphology has been successfully described using the following scaling model $[22,23]$ :

$$
\begin{gathered}
G(R)=2 \omega^{2}(R / \lambda)^{2 \alpha} \quad \text { for } R \ll \lambda \\
G(R)=2 \omega^{2} \quad \text { for } R \gg \lambda .
\end{gathered}
$$

$\lambda$ is the lateral correlation length, i.e., the distance over which the height fluctuations spread, and $R$ is the lateral scaling length. The root mean square (RMS) values of $\omega$ and $\lambda$ represent the vertical and lateral sizes of the mountains or valleys on the rough surface. Figure 5(d) shows $G(R)$ obtained from AFM measurement. The slope of the linear region represents the roughness exponent, $\alpha$, which is the result of the local surface fractal [24]. Since the surface height fluctuations have no correlation on a large scale, we can estimate $\alpha$ for the tungsten stamp on a small scale by a least squares fit of the experimental data. In the case of the ordered region (II of figure 3), $\alpha$ is 0.90 . The disordered region has a roughness exponent of 0.82 . However, $\alpha$ is calculated as 0.5 for the large area scan. The fractal dimension, $D_{\mathrm{f}}$, which is calculated from the roughness exponent with the relationship of $D_{\mathrm{f}}=3-\alpha$ $(0<\alpha<1)$, is 2.10 for the ordered and 2.18 for the disordered region. The roughness exponent of the regular mounded structure is near to unity $\left(D_{\mathrm{f}} \sim 2\right)$. Note that a higher roughness exponent means the local regular mounded structure of templates compared to a lower roughness exponent for the large area. The fluctuation of the $G(R)$ curve in the ordered region and the low roughness exponent on the large scale (figure 5(d)) are interesting but beyond the scope of this paper.

Here we defined $\lambda$ as the maximum length where the linear relationship as shown in equation (1) is maintained [22]. With increasing disorder, the roughness increases and the lateral correlation length also increases to about $37.0 \mathrm{~nm}$. But in the ordered region, $\lambda$ is $32.0 \mathrm{~nm}$ and it is close to that of the whole image $(\lambda=32.8 \mathrm{~nm})$. This means that the disorder is a local phenomena due to the local deformation of the corrugation surface as explained in the previous section. On a large scale, the surface looks flat without correlated fluctuations 
Fabrication of a tungsten master stamp using self-ordered porous alumina

and the lateral correlation length obtained in the height-height correlation function is consistent with the cut-off frequency of the FFT analysis.

\section{Conclusion}

Highly ordered porous alumina was obtained without the use of any sacrificial layer by using an easily fabricated nanoimprint stamp for pore nucleation. The stamp consists of tungsten nanodots with a height of $15 \mathrm{~nm}$, and was prepared by self-assembly of a porous alumina template and subsequent DC sputtering. The hexagonally arranged pattern with $100 \mathrm{~nm}$ lattice constant was embossed onto an electropolished aluminium sample by using a pneumatic oil press with a pressure of $15 \mathrm{kN} \mathrm{cm}^{-2}$. We were able to obtain highly ordered porous alumina by anodizing such prepatterned aluminium. Previously, such high quality pore nucleation was achievable only after removing the first formed oxide (sacrificial layer) with a thickness of $100 \mu \mathrm{m}$ in the twostep anodization method. In addition, fractal calculations were performed to evaluate the degree of order of nanodots on the tungsten stamp, and these showed that the results are in good agreement with theory. The tungsten nanoimprint lithography allows us to cost-effectively prepare highly ordered porous alumina. We expect this technique of stamp definition to be immediately applicable for defining highly ordered porous alumina thin films on semiconductors, which have been hard to obtain due to the limit on the evaporated thickness.

\section{References}

[1] Martin C R 1994 Science 2661961

[2] Steinhart M, Wendorff J H, Greiner A, Wehrspohn R B, Nielsch K, Schilling J, Choi J and Gösele U 2002 Science 2961997
[3] Choi J, Sauer G, Nielsch K, Wehrspohn R B and Gösele U 2003 Chem. Mater. 15776

[4] Masuda H and Fukuda K 1996 Science 2681466

[5] Li A P, Müller F, Birner A, Nielsch K and Gösele U 1998 J. Appl. Phys. 846023

[6] Masuda H, Yamada H, Satoh M, Asoh H, Nakao M and Tamamura T 1997 Appl. Phys. Lett. 712770

[7] Li A P, Müller F and Gösele U 2000 Electrochem. Solid State Lett. 3131

[8] Mikulskas I, Juodkazis S, Tomasiunas R and Dumas J G 2001 Adv. Mater. 131574

[9] Choi J, Nielsch K, Reiche M, Wehrspohn R B and Gösele U 2003 J. Vac. Sci. Technol. B 21763

[10] Liu N W, Datta A, Liu C Y and Wang Y L 2003 Appl. Phys. Lett. 821281

[11] Sun Z J and Kim H K 2002 Appl. Phys. Lett. 813458

[12] Masuda H, Yotsuya M, Asano M, Nishio K, Nakao M, Yokoo A and Tamamura T 2001 Appl. Phys. Lett. 78826

[13] Choi J, Sauer G, Göring P, Nielsch K, Wehrspohn R B and Gösele U 2003 J. Mater. Chem. 131100

[14] Rabin O, Herz P R, Lin Y M, Akinwande A I, Cronin S B and Dresselhaus M S 2003 Adv. Funct. Mater. 13631

[15] Choi J, Wehrspohn R B and Gösele U 2003 Adv. Mater. 15 1531

[16] Fournier-Bidoz S, Kitaev V, Routkevitch D, Manners I and Ozin G A 2004 Adv. Mater. 162193

[17] www.matweb.com

[18] Choi J, Wehrspohn R B, Lee J and Gösele U 2004 Electrochim. Acta $\mathbf{4 9} 2645$

[19] Li F Y, Zhang L and Metzger R M 1998 Chem. Mater. 102470

[20] Liu C Y, Datta A and Wang Y L 2001 Appl. Phys. Lett. 78120

[21] Choi J, Schilling J, Nielsch K, Hillebrand R, Reiche M, Wehrspohn R B and Gösele U 2002 Mater. Res. Soc. Symp. Proc. 722 L5.2

[22] Tong W M and Williams R S 1994 Annu. Rev. Phys. Chem. 45 401

[23] Family F and Vicsek T 1985 J. Phys. A: Math. Gen. 18 L75

[24] Vazquez L, Salvarezza R C, Ocon P, Herrasti P, Vara J M and Arvia A J 1994 Phys. Rev. E 491507 